\title{
TR-PIV in highly pulsatile flow: pulsation frequency and wake dynamics case study
}

\author{
I. A. Carr ${ }^{1}$, M. W. Plesniak ${ }^{2 *}$ \\ ${ }^{1}$ Division of Applied Mechanics, Office of Science and Engineering Laboratories, Center for Devices and \\ Radiological Health, US Food and Drug Administration, Silver Spring, MD, USA \\ ${ }^{2}$ Department of Mechanical and Aerospace Engineering, George Washington University, Washington, DC, \\ USA \\ *plesniak@gwu.edu
}

\begin{abstract}
Experimental study of highly pulsatile flows presents a number of challenges, primarily the inherently large dynamic range of velocities. Herein, we use time-resolved particle image velocimetry processed with a technique known as pyramid sum-of-correlation to study highly pulsatile flow around a surface-mounted hemisphere. The frequency of pulsation is varied from low-frequency, quasi-steady pulsation to high frequency pulsation. We present a conceptual overview of the wake regimes observed and compare the flow physics of the high-frequency case to that of a vortex ring produced by a single impulse of fluid.
\end{abstract}

\section{Introduction}

Surface-mounted obstacles produce a plethora of flow phenomena. Herein we use time-resolved particle image velocimetry (TR-PIV) to study the effects of a highly pulsatile freestream with variable pulsation frequency on the wake dynamics of a surface-mounted hemisphere. Given the inherently large dynamic range of velocities in this flow field, both variation due to freestream pulsation and wake-to-freestream differential, attaining TR-PIV data requires the use of multiple frames during correlation.

In this study we discuss the TR-PIV methods used and their applicability to highly pulsatile flow, as well as changes in the wake dynamics with changing freestream pulsatile frequency. These results are discussed in more detail in Carr et al. (2020).

\section{Methods}

The studies were conducted in a small-scale, low-speed, pulsatile wind tunnel designed and constructed for these experiments, shown in Figure 1. The pulsatile inflow velocity profile produced by the pulsatility generator depicted in Figure 2 is roughly sinusoidal, varying from $\operatorname{Re}=0$ to 2000, with an average Re of 1000, as shown in Figure 3 and Figure 4. The reduced frequency of pulsation, $k$, varied from 0.01 to 0.1 as is shown in Figure 3. T The TR-PIV system was comprised of an Photonics Ind. Nd:YLF laser $(527 \mathrm{~nm}, 10 \mathrm{kHz})$ and an IDT MotionPro Y7 high speed camera (12k FPS), and laser sheet forming optics from Thorlabs.

The PIV processing was performed in LaVision DaVis 8.4.0 software. To compute the flow velocity throughout the pulsatile cycle, an averaging process termed the pyramid sum-of-correlation from Sciacchitano et al. (2012) was employed. This technique is particularly useful for flows with a large dynamic range of velocities. Highly pulsatile flow such as the one studied herein is a particularly informative showcase of this technique.

Figure 4 shows the phases of the inflow waveform we will be using to examine the flow field. The phases shaded in red will be referred to as "accelerating" and those in blue as "decelerating". From the velocity

\footnotetext{
${ }^{1} k=R f / \bar{u}$ where $R$ is the hemisphere radius, $f$ is the frequency of the pulsatility in $\mathrm{Hz}, \bar{u}$ is the average streamwise velocity, and $\mathrm{P}$ is the period.
} 


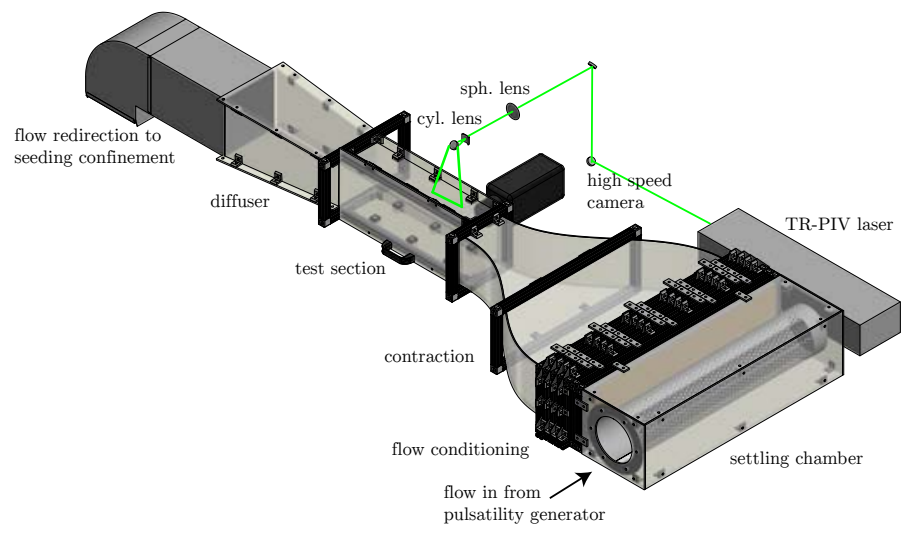

Figure 1: Schematic of low speed pulsatile wind tunnel with TR-PIV setup.

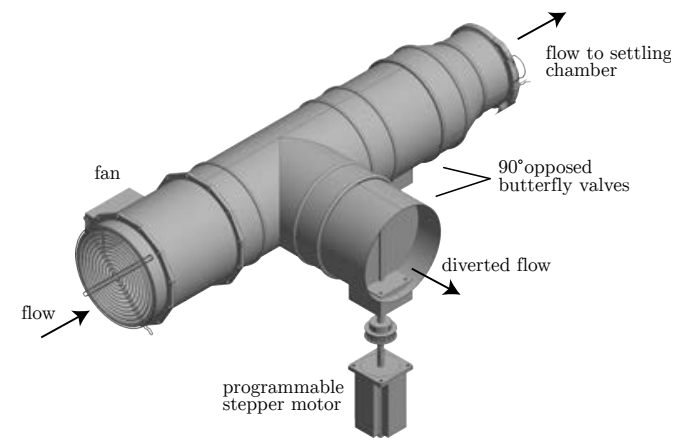

Figure 2: Schematic of butterfly valvebased pulsatility generator

variation shown in Figure 4 it is clear that traditional PIV would require tuning the capture parameters for each phase to obtain usable data - significantly increasing experiment time.

Using TR-PIV in combination with the pyramid sum-of-correlation technique considerably reduces the complexity and time required to perform these experiments. Instead of changing the time between frames, $d t$, corresponding to the value of freestream velocity at each phase, we were able to resolve the flow field keeping the time between frames at a constant $d t=164 \mu \mathrm{s}$ - which equates to $6 \mathrm{kHz}$ framerate on the high speed camera.

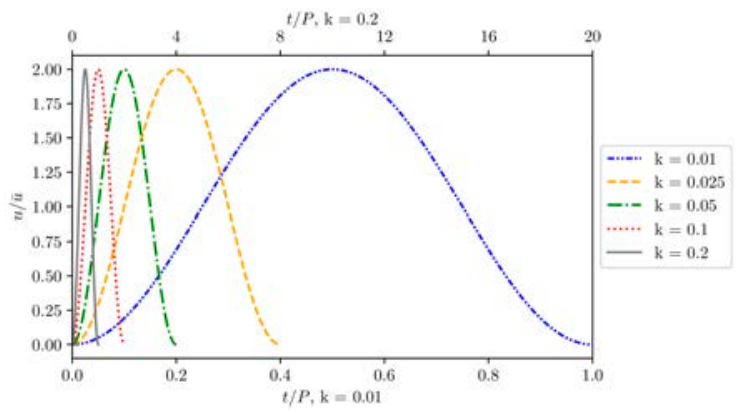

Figure 3: Idealized representation of the sinusoidal inflow waveforms of varying reduced frequency, $k$.

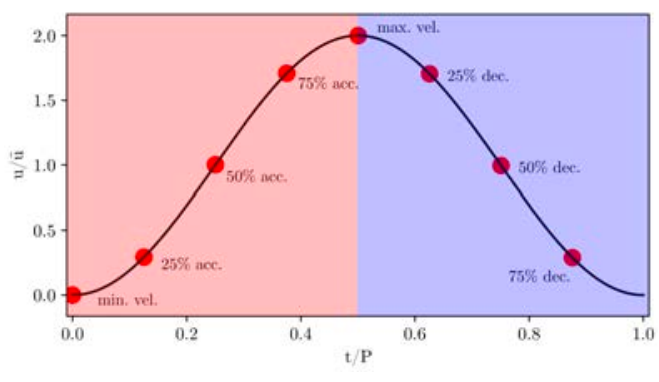

Figure 4: A pulsatile inflow waveform with markers indicating the position of the data displayed in Figure 5. The region shaded red indicates the "accelerating" phases and blue indicates the "decelerating" phases.

Using the aforementioned techniques, we captured data at the phases shown in Figure 4 for each inflow waveforms shown in Figure 3 with the exception of the highest value, $k=0.2$, due to limitations in the pulsatility generator.

\section{Results and Discussion}

Varying $k$ results in regime changes in the wake dynamics. Figure 5 shows four velocity fields taken throughout the inflow cycle for three of the values of $k$ studied. The four phases are spaced evenly throughout the inflow profile as shown in Figure 4. Near the low end of the reduced frequency range the wake resembles that of a hemisphere in steady flow at the equivalent Re (Acarlar and Smith, 1987). At the high end the wake dynamics are phased locked to the freestream forcing, producing only a single vortex structure per cycle.

Figures $5 \mathrm{a}$-d represent the lowest value of $k$, in which hairpin vortices shed from a shear layer (highlighted in red) and there are no significant organized flow structures near minimum velocity (Figure 5a). The hairpin vortices are produced from a Kelvin-Helmholtz instability near the downstream extent of the 

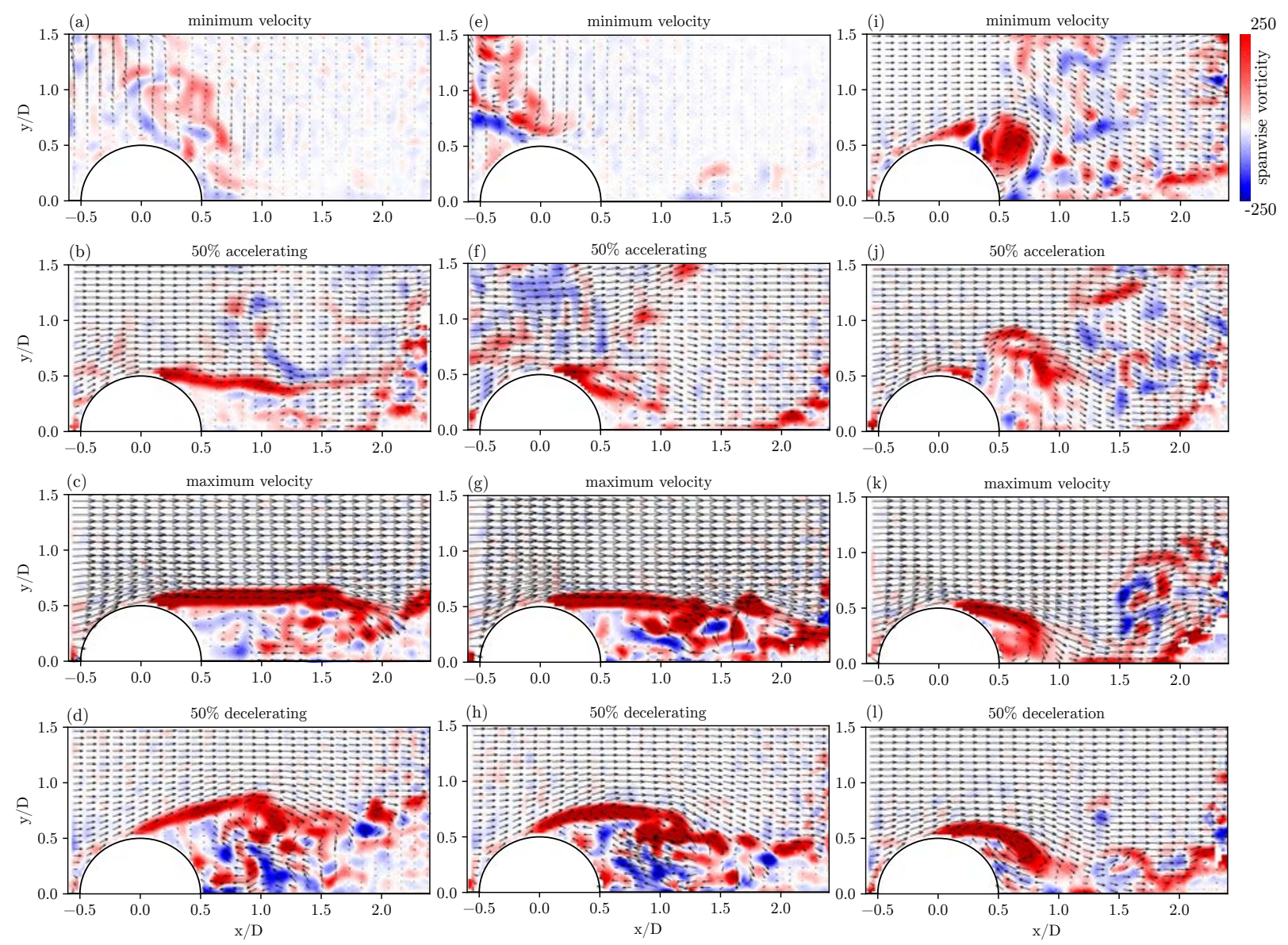

Figure 5: Centerline velocity fields with spanwise vorticity contours from four positions in the pulsatile inflow cycle: maximum and minimum velocity along with times in the middle of inflow acceleration and deceleration. Three of the values of $k$ studied are shown. The axes are normalized by the diameter of the hemisphere, $D$.

shear layer. For $k=0.01$ the wake resembles that of a hemisphere in steady flow with the exception of the decelerating phases in which the shear layer lifts away from the surface and breaks up into small scale turbulence.

Figures 5e-h represents a transitional case which shares some characteristics with both the high and low cases. While there is a shear layer, the presence of the hairpin vortices is significantly reduced. In this case there is less time for the shear to develop and shed hairpins resulting in fewer, if any, hairpins released before deceleration begins and the shear layer is disrupted.

Figures 5-1 represents the high end of the range of $k$ in which the wake is dominated by a single arch vortex which is phase-locked with the pulsatile freestream. The shear layer begins forming during acceleration and continues through maximum velocity. The time for the formation to take place is so short, the shear layer only extends roughly $1 \mathrm{D}$ downstream before it is rolled up into a single arch vortex during inflow deceleration.

Of most interest is the transition from a quasi-steady case in which the wake is characterized by a shear layer which sheds hairpin vortices downstream, to a single arch vortex produced once per pulse. To more clearly communicate the progression of wake regimes with increasing reduced frequency, Figure 6 is a series of schematic representations of the primary wake structures observed at each of the reduced frequency.

Progressing from $k=0.01$ to $k=0.2$, the wake of the hemisphere changes from a quasi-steady case which is largely made up of the same structures seen in steady flow, to a highly pulsatile case in which each pulse produces its own vortex structure. As represented in Figure 6 and in Figure 5-1, the transition from a 


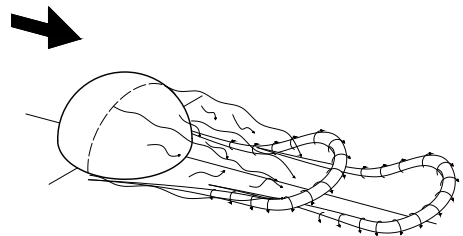

turbulent wake \& $\mathrm{K}-\mathrm{H}$ shedding

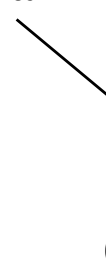

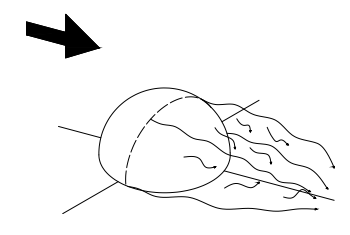

turbulent wake only

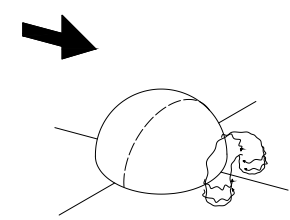

ragged, phase-locked single structure

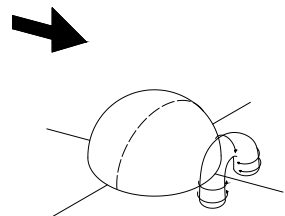

smooth, phase-locked single structure

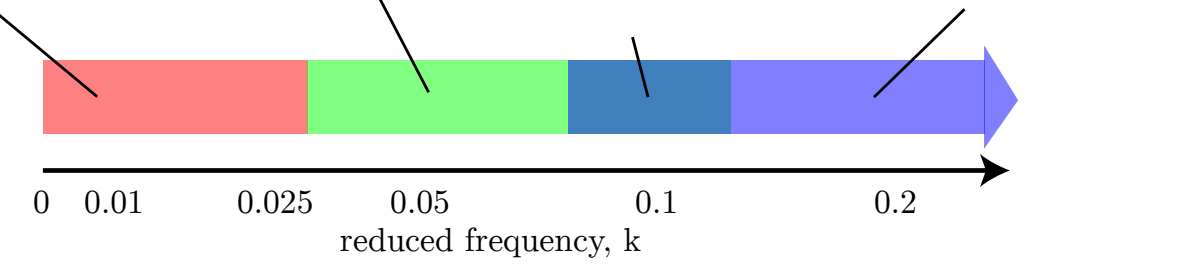

Figure 6: Schematic representations of the primary wake structures with increasing reduced frequency.

wake resembling steady flow to a single flow structure per pulse occurs near $k=0.1$. At $k$ higher than 0.1 arch vortices will continuously form with each pulse though their intensity and size may change. At $k$ lower than 0.01 , the wake will continuously approach a steady flow configuration.

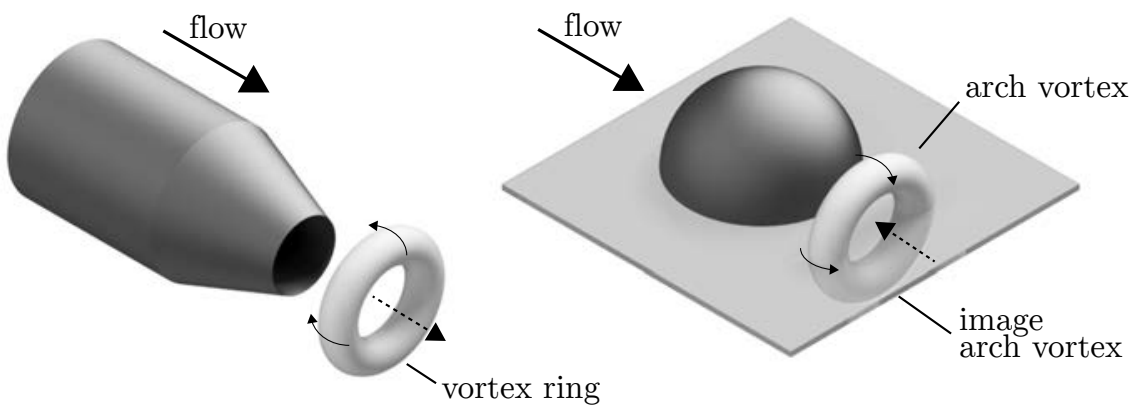

Figure 7: Conceptual comparison of a vortex ring produced with the traditional, sharp-edged piston-driven vortex generator and the arch vortex in the wake of a hemisphere in pulsatile flow.

In these higher frequency cases, $k>0.1$, the pulsation so dominates the flow physics that they resemble that of a single impulse of fluid rather than a continuously flowing freestream. To demonstrate this, we draw the analogy between the arch vortex and a vortex ring produced from a sharp-edged piston-driven vortex generator, as depicted in Figure 7. Vortex rings have been extensively studied and in many of those studies the vortex ring is formed at the outlet of a sharp edged piston vortex generator. The vortex ring is formed and propagates in the same direction as the fluid ejection. At the high end of $k$, the arch vortex in the wake of the hemisphere is produced with the same mechanisms. Interestingly, in this configuration, the direction of propagation in the arch vortex is counter to the freestream flow. This is, in part, what accounts for some of the vortex dynamics seen in deceleration - a more in-depth discussion can be found in Carr and Plesniak (2016).

The series of flow fields measured herein has an inherently wide range of velocities both within one phase and throughout the pulsatile cycle. While traditional, more labor intensive techniques would allow measurement of these flow fields, the combination of TR-PIV and the pyramid sum-of-correlation techniques made resolving these flow fields significantly quicker and less prone to experimental error.

\section{Conclusions}

In this study we investigated the effects of highly pulsatile flow with different pulsation frequencies on the wake dynamics of a surface-mounted hemisphere. To handle the inherently large dynamic range of velocities 
in these flow fields we used TR-PIV and processed the data using the pyramid sum-of-correlation technique. These techniques produced high quality velocity data from which we can observe the changing wake regimes with increasing $k$. To better conceptualize these regimes we present schematic depictions of the primary flow structures in four regimes. We also compare the wake dynamics at high values of $k$ to that of a vortex ring ejected from a piston driven vortex generator. This conceptual overview provides a framework for understanding the interaction between pulsatile flow and surface-mounted obstacles. Moreover, it provides a useful example of employing TR-PIV and pyramid sum-of-correlation processing and the utility of those techniques.

\section{References}

Acarlar MS and Smith CR (1987) Study of hairpin vortices in a laminar boundary layer: Part 2. hairpin vortices generated by fluid injection. Journal of Fluid Mechanics 175

Carr IA, Beratlis N, Balaras E, and Plesniak MW (2020) Effects of highly pulsatile inflow frequency on surface-mounted bluff body wakes. Journal of Fluid Mechanics 904

Carr IA and Plesniak MW (2016) Three-dimensional flow separation over a surface-mounted hemisphere in pulsatile flow. Experiments in Fluids 57:9

Sciacchitano A, Scarano F, and Wieneke B (2012) Multi-frame pyramid correlation for time-resolved PIV. Experiments in Fluids 53:1087-1105 